This is a self-archived version of an original article. This version may differ from the original in pagination and typographic details.

Author(s): Eskelinen, Matti; Hämäläinen, Jyri

Title: Dangers of Demosaicing : Confusion From Correlation

Year: 2019

Version: Accepted version (Final draft)

Copyright: (C) IEEE, 2019.

Rights: In Copyright

Rights url: http://rightsstatements.org/page//nC/1.0/?language=en

Please cite the original version:

Eskelinen, M., \& Hämäläinen, J. (2019). Dangers of Demosaicing : Confusion From Correlation. In WHISPERS 2018 : 9th Workshop on Hyperspectral Image and Signal Processing, Evolution in Remote Sensing. IEEE. https://doi.org/10.1109/WHISPERS.2018.8747204 


\title{
DANGERS OF DEMOSAICING: CONFUSION FROM CORRELATION
}

\author{
Matti A. Eskelinen, Jyri Hämäläinen \\ University of Jyväskylä \\ Faculty of Information Technology
}

\begin{abstract}
Images from colour sensors using Bayer filter arrays require demosaicing before viewing or further analysis. Advanced demosaicing methods use empirical knowledge of inter-channel correlations to reduce interpolation artefacts in the resulting images. These inter-channel correlations are however different for standard RGB cameras and hyperspectral imagers using colour sensors with added narrow-band spectral filtering.

We study the effects of conventional demosaicing methods on hyperspectral images with a dataset originally collected without a colour filter array. We find that using advanced methods instead of bilinear interpolation results in an overall increase of $9-14 \%$ in absolute error and a decrease of $1-3 \%$ in PSNR, but also observed a decrease in MSE of $11-13 \%$.

For the corresponding RGB images, the advanced methods improved fidelity as expected. The results also demonstrate that the reconstruction methods that take advantage of correlation transport noise present in a single component to other reconstructed layers.
\end{abstract}

Index Terms - Hyperspectral imaging, Fabry-Perot, Instruments, Bayer pattern, Colour filter array (CFA) interpolation, Demosaicing, Algorithms

\section{INTRODUCTION}

Modern hyperspectral "snapshot" imagers based on FabryPerot interferometers as their filtering technique can use either monochromatic or Bayer-filtered sensors as their imaging component. The latter case allows imaging of multiple narrow wavebands with a single exposure, as the radiances for each single wavelength can still be reconstructed based on the known quantum efficiencies of the different pixel filters for the given wavelengths [1]. However, since only a measurement of a single colour filter is available at each location, interpolation (or demosaicing) has to be used to approximate the other filter responses at each location in order to solve for spectral radiances.

This research is in part funded by the Jane and Aatos Erkko foundation (grant 170015) and in part by the Finnish Funding Agency for Innovation (TEKES) (grant 1711/31/2016).
Much work has been put into the development of demosaicing methods due to the prevalence of Bayer filters in digital consumer cameras. Reducing interpolation artefacts without sacrificing too much computational efficiency is a problem that has provided us with a wealth of existing literature. Many approaches are based on utilising the correlation of the luminosity and chromaticity in natural images to improve the SNR of the reconstruction $[2,3]$. We give a short summary of the methods under comparison in section 2.

The correlations are partially reliant on the fact that the colour filters of the typical Bayer filter array are approximating human vision and thus have overlapping transmittances across a range of wavelengths. However, for hyperspectral imagers using the Bayer filter reconstruction technique with narrow-band filtering, the correlation between the signals of different colour filters is dependent on the wavelengths transmitted by the narrow-band filter. While demosaicing methods have been developed also for imagers with multispectral filter configurations [4], there is little literature on the applicability of existing methods in use cases related to spectral imaging.

To study the effect this discrepancy has on the resulting data, we construct a benchmark for the radiance reconstruction using radiances from data taken using a more traditional filter system. The procedures used are detailed in section 3 . We use the benchmark to compare the more advanced methods against bilinear filtering - which does not rely on correlations - and present our findings in section 4 .

\section{DEMOSAICING METHODS}

A CFA image is a matrix composed of three (or more) different kinds of pixels in a given pattern. An example of a typical Bayer "RGGB" pattern is given in figure 1, with R, G and B referring to the red, green and blue filters respectively. Demosaicing entails the computation of a full-size layer of values for each of the component types, with the missing values in other locations filled in using interpolation.

We compared three demosaicing methods that had readily available software implementations, specifically those implemented by the "Colour - demosaicing" Python library [5]. What follows is a brief summary of each, for the readers disinclined to wade through the references. 


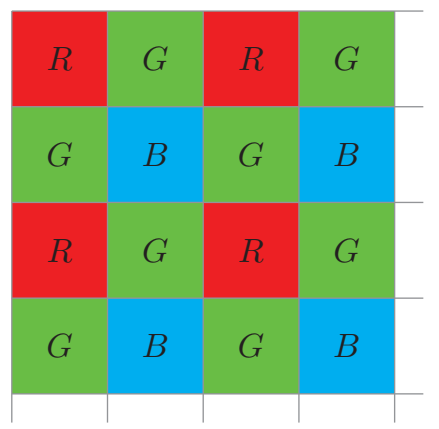

Fig. 1. Example of an RGGB Bayer filter pattern.

\subsection{Bilinear interpolation}

Bilinear interpolation fills each missing value with the average of its nearest neighbours. Interpolation is done on each component plane independently, without any assumptions of correlations between the components. While it can be implemented highly efficiently using a discrete convolution, it causes highly visible artefacts in the resulting image. [6]

\subsection{Malvar (2004)}

The method of Malvar, He and Cutler [2] is similar to the simple bilinear interpolation, but adds to each interpolated value a correction based on the estimated gradients of the other components, depending on the location of the pixel with respect to the pattern. Like bilinear interpolation, it is also implemented as a linear convolution, making it computationally efficient. It is familiar to many as the default demosaicing method in the MATLAB Image Processing Toolbox [7].

The precise weights used for the convolution implementation of their algorithm are the result of an experimental fit to find the best reconstruction of the Kodak colour image dataset, with a constraint on the divisibility to find an efficient binary representation.

\subsection{Menon (2007)}

The method of Menon, Adriani and Calvagno [3] utilizes directional filtering and a posteriori selection of the best interpolation of the green component. The other colour components in the green locations are reconstructed using bilinear interpolation.

The directional information in the green layer is then used to help reconstruct the colour difference layers $(R-B)$ and $(B-G)$, which are then used to recreate the red and blue components at other locations, along with corrections based on frequency filtering. The approach relies heavily on the correlation between the channels, and is roughly 3-5 times more computationally intensive then the bilinear interpolation.

\section{TEST METHODOLOGY}

\subsection{Radiance dataset}

In order to study the effect of demosaicing on hyperspectral images we needed data with a similar structure to the output of Fabry-Perot imagers, but which has been collected without a Bayer filter. The data could then be converted to a mosaic trough simple omission and the results of demosaicing could be reliably compared to the original without having to worry about any systematic error arising from previous reconstruction methods.

We decided to use as the base data the publicly available radiance images of natural scenes by Foster, Nascimento and Amano [8], which were acquired using a full-frame imager with a tunable filter and a monochromatic (non-array) sensor. The characteristics of the natural scenes also resemble those of the Kodak dataset often used in demosaicing studies, with varying edge features and spectral signatures. Each of the thirty images had 33 radiance bands evenly distributed between wavelengths of $400 \mathrm{~nm}$ and $720 \mathrm{~nm}$ with a band width of $10 \mathrm{~nm}$.

The dataset also included an RGB image of each scene reconstructed from the radiance using the CIE standard observers. These were used as-is to test the performance of the methods for this dataset.

\subsection{Mosaic construction}

For each of the 33 band radiance cubes in the dataset, we constructed a corresponding mosaic cube of 11 CFA images with an RGGB pattern with each image having its R, G and B pixels sampled from different wavelength bands of the cube. The wavelengths used for each mosaic layer are listed in table 1 . The choice of the specific pattern was arbitrary. While we did not verify it experimentally, the different orderings of the colour components should produce equivalent results since none of our fidelity measures use directional quantities which might change under different patterns.

The wavelengths were selected by simply dividing the set of radiance bands into three equal parts, and using the longer wavelengths for the red, the medium for green and shorter for blue in consecutive triplets, such that no triplet contained bands near each other in wavelength. Since there is no sensorinduced correlation in the different radiance values, the ordering of the bands should not matter; We opted to match the wavelengths roughly to the colour components they affect the most. This way any correlations between the radiances present in the natural scenes due to spectral properties of the specific scene should be roughly similar to those in true-colour images.

This method of construction approximates the structure of data from a spectral imager using a scanning Fabry-Perot interferometer with three of the transmission peaks in the range 
Table 1. Wavelength bands from of the base image used for the different pixels in each mosaic layer of the corresponding test dataset.

\begin{tabular}{rrrr}
$\#$ & $\lambda_{B}$ & $\lambda_{G}$ & $\lambda_{R}$ \\
\hline 1 & 400 & 510 & 620 \\
2 & 410 & 520 & 630 \\
3 & 420 & 530 & 640 \\
4 & 430 & 540 & 650 \\
5 & 440 & 550 & 660 \\
6 & 450 & 560 & 670 \\
7 & 460 & 570 & 680 \\
8 & 470 & 580 & 690 \\
9 & 480 & 590 & 700 \\
10 & 490 & 600 & 710 \\
11 & 500 & 610 & 720 \\
\hline
\end{tabular}

of sensor sensitivity at once. It is simplified in that the different transmission peaks are not mixed at all in the R, G and $B$ pixels unlike in a real imager, where the response of each pixel to the peaks would be dependent on the wavelength.

We decided against the modelling of a more realistic situation for this study, since the choices needed for the construction of the more realistic mosaics would complicate interpretation of any results and proper analysis would have taken more time then was available for the preparation of this paper.

\subsection{Fidelity criteria}

Since perceptual metrics based on human vision do not make sense for the radiance data directly, we consider only objective fidelity measures based on the numerical difference of the original and the reconstructed image. Following [6], we measure the fidelity of the demosaiced images with $X \times Y$ pixels for each band $b$ using the mean absolute error,

$$
\operatorname{MAE}_{b}\left(I, I^{\prime}\right)=\frac{1}{X Y} \sum_{x=0}^{X-1} \sum_{y=0}^{Y-1}\left|I_{x, y}-I_{x, y}^{\prime}\right|
$$

mean square error,

$$
\operatorname{MSE}_{b}\left(I, I^{\prime}\right)=\frac{1}{X Y} \sum_{x=0}^{X-1} \sum_{y=0}^{Y-1}\left(I_{x, y}-I_{x, y}^{\prime}\right)^{2}
$$

and peak signal-to-noise ratio

$$
\operatorname{PSNR}_{b}\left(I, I^{\prime}\right)=10 \cdot \log _{10}\left(\frac{\max \left(I_{b}\right)^{2}}{\operatorname{MSE}_{b}\left(I, I^{\prime}\right)}\right)
$$

with the original radiance images $I$ and the reconstructions $I^{\prime}$. The same metrics were also calculated for the RGB images and their reconstructions using colour components in place of radiance bands.
Table 2. Average fidelities of the Malvar and Menon methods relative to bilinear filtering on the radiance and RGB images.

\begin{tabular}{rllll} 
& \multicolumn{2}{c}{ Radiance } & \multicolumn{2}{c}{ RGB } \\
\cline { 2 - 5 } & Malvar & Menon & Malvar & Menon \\
\hline MAE & 1.09 & 1.14 & 0.97 & 1.15 \\
MSE & 0.79 & 0.77 & 0.54 & 0.66 \\
PSNR & 0.99 & 0.97 & 1.15 & 1.11 \\
\hline
\end{tabular}

The three fidelity measures were averaged over the dataset for each band and ordered to match the structure of the original radiance data. The metrics for each method were also divided by the corresponding metric for bilinear interpolation to get a touch on their relative performance. Corresponding values were also calculated for the RGB reconstructions to verify that the methods perform as expected for the kind of images they were designed for.

\section{RESULTS}

Figures 2, 3 and 4 show the MAE, MSE and PSNR for the different methods. The metrics were calculated for each wavelength band and averaged over the test dataset. The absolute values show a significantly worse reconstruction for the first band in all the cases. This is due to the high noisiness of the data in the 400nm band, which was known for the dataset and confirmed by us with a visual inspection. This noisiness carries over to the relative metrics for the Malvar and Menon methods, in that the reconstruction of 510nm and $620 \mathrm{~nm}$ bands included in the same mosaics with the noisy waveband suffer with the methods using correlation. This was also visually apparent in the reconstruction of the given bands.

The relative fidelities of the Malvar and Menon methods over all the bands are presented in table 2 for both the radiance and RGB reconstructions. For comparison, Malvar, He and Cutler report an increase of $5.68 \mathrm{~dB}$ in PSNR for their method over the bilinear interpolation [2], while Menon, Adriani and Calvagno claim an increase of $9.69 \mathrm{~dB}$ [3]. Their tests were conducted respectively on sets of 15 and 20 RGB colour images from the Kodak dataset.

\section{CONCLUSIONS AND FUTURE WORK}

The relative fidelity measures demonstrate that introducing a correlation-based correction in the interpolation also has the side effect of carrying over any noise present in the bands to the other interpolated layers, which is to be expected. Similar effects are present in the reconstruction of radiance measurements from actual Fabry-Perot hyperspectral imagers in the bands where correlation is introduced by the mixing of the different narrow-band radiances on the sensors (though dif- 


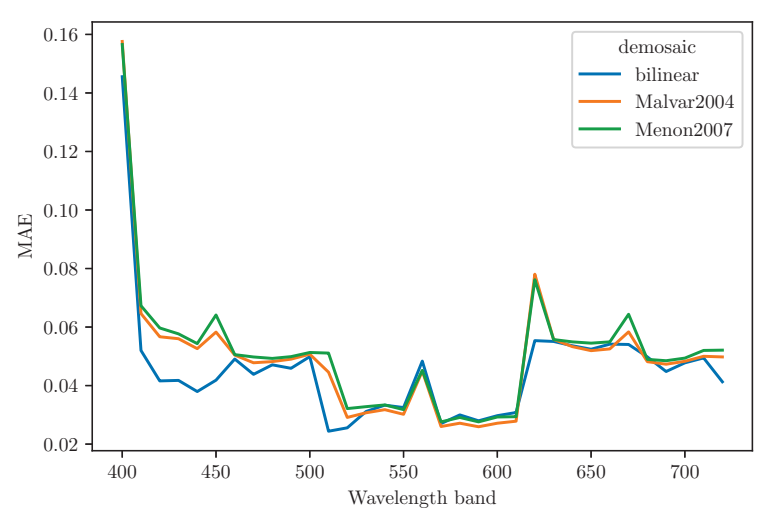

(a)

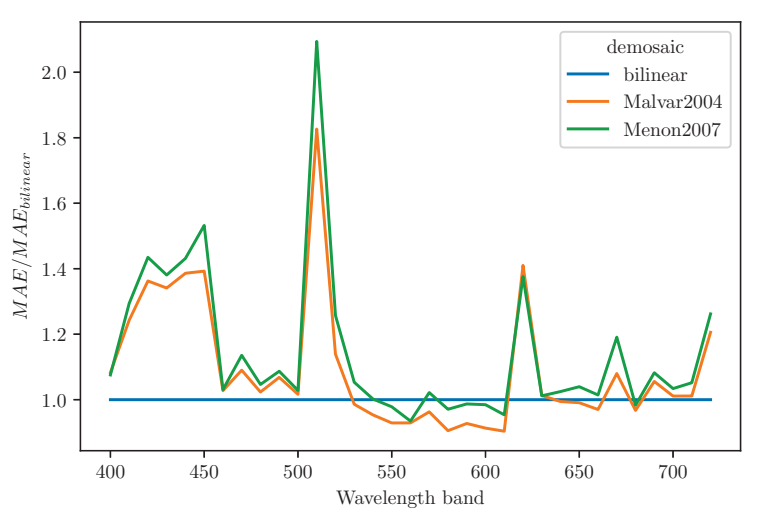

(b)

Fig. 2. (a) Average MAE over the full dataset for each wavelength band. (b) Average MAE compared to the bilinear interpolation.

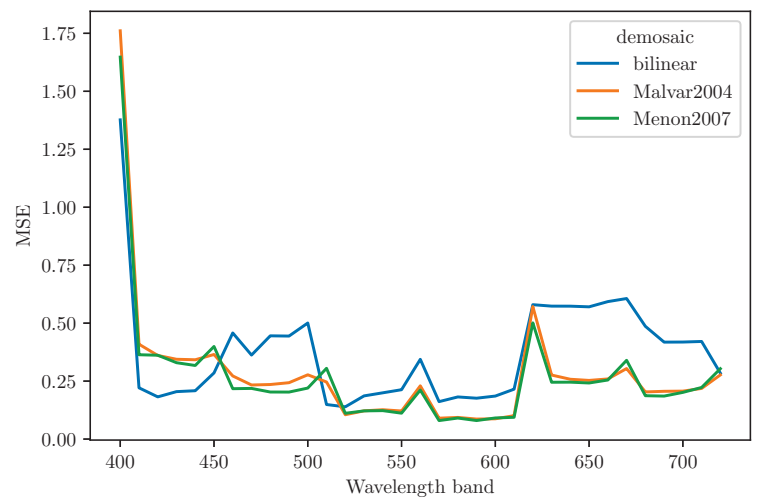

(a)

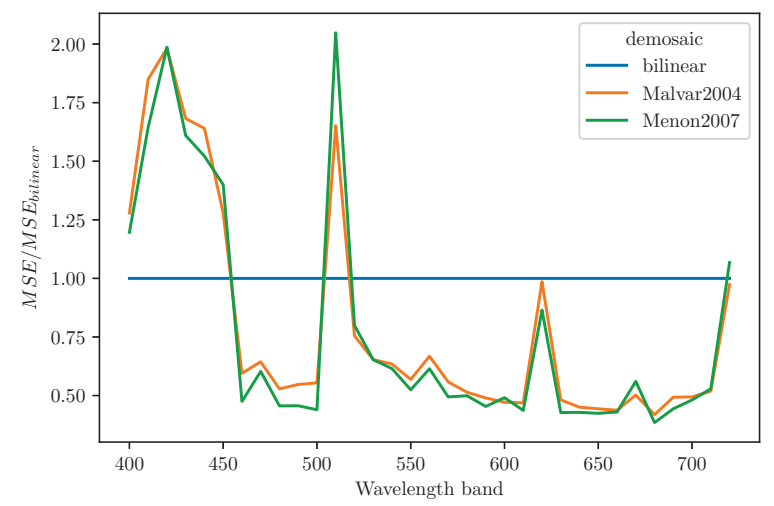

(b)

Fig. 3. (a) Average MSE over the full dataset for each wavelength band. (b) Average MSE compared to the bilinear interpolation.

ferent in magnitude to the usual correlation of the $R, G$ and $B$ pixels).

All the measures also show generally worse performance for the Malvar and Menon methods in the noisy band, which is most likely due to their use of gradients (which are generally sensitive to noise). However, the advanced methods perform slightly better in the bands with lower noise. While we did not comprehensively study the effect of the ordering of the bands, some tests ran after the calculation of the main results suggest that the ordering does not significantly change the overall performance, although some differences arise on the single bands. This suggests that the methods are not very sensitive to the chromatic correlations in the natural scenes, but that differences arise based on the exact band used for the better sampled component (usually the green one).

The relative fidelities in table 2 show that the overall fi- delity of the advanced reconstruction is decreased by a few percent compared to the bilinear interpolation. The results on reconstructed RGB images show that when the assumptions of the algorithms are fulfilled, the fidelity of the reconstruction should increase significantly for this specific dataset.

While we did not evaluate any perceptual metrics, visual inspection of some of the reconstructions suggested that while the advanced methods generally did not cause noticeable degradation in the radiance images, localised artefacts were generated for certain materials (spectral signatures) in the scenes. More comprehensive study comparing spectral differences using e.g. spectral angle or correlation would be needed to quantify these effects. 


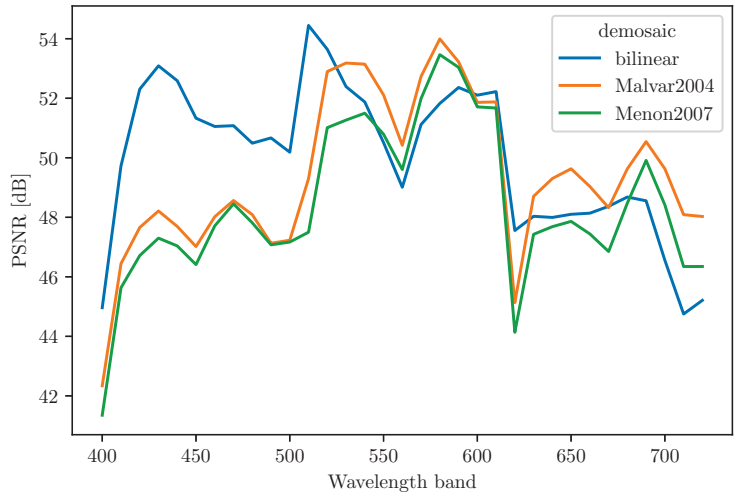

(a)

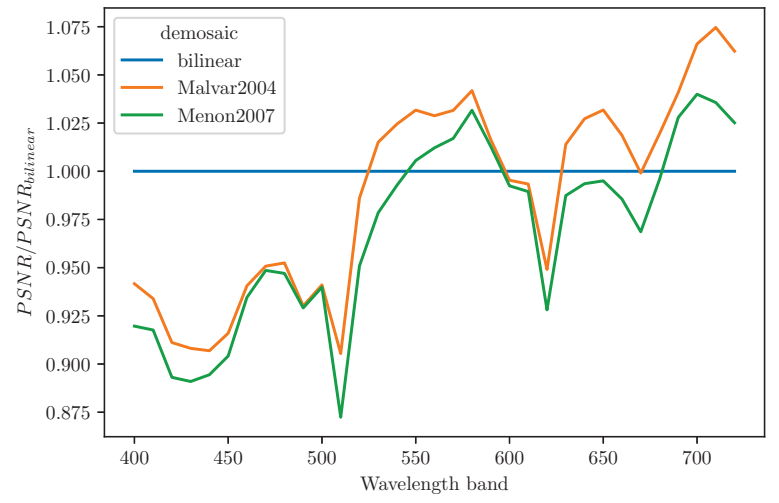

(b)

Fig. 4. (a) Average PSNR over the full dataset for each wavelength band. (b) Average PSNR compared to the bilinear interpolation.

\section{REFERENCES}

[1] Heikki Saari, Ilkka Pölönen, Heikki Salo, Eija Honkavaara, Teemu Hakala, Christer Holmlund, Jussi Mäkynen, Rami Mannila, Tapani Antila, and Altti Akujärvi, "Miniaturized hyperspectral imager calibration and UAV flight campaigns," in Proc. SPIE. Oct. 2013, vol. 8889 , p. 888910 , International Society for Optics and Photonics.

[2] Henrique S Malvar, Li-Wei He, Ross Cutler, and One Microsoft Way, "High-Quality Linear Interpolation for Demosaicing of Bayer-Patterned Color Images," in International Conference of Acoustic, Speech and Signal Processing. may 2004, pp. 5-8, Institute of Electrical and Electronics Engineers, Inc.

[3] Daniele Menon, Stefano Andriani, and Giancarlo Calvagno, "Demosaicing With Directional Filtering and a posteriori Decision," IEEE Transactions on Image Processing, vol. 16, no. 1, pp. 132-141, jan 2007.
[4] Pierre-Jean Lapray, Xingbo Wang, Jean-Baptiste Thomas, and Pierre Gouton, "Multispectral Filter Arrays: Recent Advances and Practical Implementation," Sensors, vol. 14, no. 11, pp. 21626-21659, Nov. 2014.

[5] The Colour Developers, "Colour - Demosaicing," Online, 2018, Software available at http://colourscience.org/colour-demosaicing/.

[6] O. Losson, L. Macaire, and Y. Yang, "Comparison of Color Demosaicing Methods," in Advances in Imaging and Electron Physics, vol. 162, pp. 173-265. 2010.

[7] Mathworks Nordic, "Convert Bayer pattern encoded image to truecolor image - MATLAB demosaic," Online, https://se.mathworks.com/help/images/ref/demosaic.html.

[8] Sérgio M. C. Nascimento, Kinjiro Amano, and David H. Foster, "Spatial distributions of local illumination color in natural scenes," Vision Research, vol. 120, pp. 39-44, Mar. 2016. 